\title{
Strategic water management for reliable mine water supply under dynamical climates
}

\author{
$\underline{\text { Lei Gao }}^{\text {a }}$, Jeffery D. Connor ${ }^{b}$, Damian Barrett ${ }^{c}$, Yun Chen ${ }^{b}$, and Xiangfeng Zhang ${ }^{a, d}$ \\ ${ }^{a}$ CSIRO Water for a Healthy Country National Research Flagship, CSIRO Land and Water, Glen Osmond, \\ South Australia, Australia \\ ${ }^{b}$ CSIRO Ecosystem Sciences, Glen Osmond, South Australia, Australia \\ ${ }^{c}$ CSIRO Water for a Healthy Country National Research Flagship, CSIRO Land and Water, Canberra, \\ Australian Capital Territory, Australia \\ ${ }^{d}$ School of Electrical Engineering, Shanghai Dianji University, Minhang District, Shanghai, China \\ Email addresses: lei.gao@csiro.au
}

\begin{abstract}
Ensuring water security is essential for a broad range of mining activities, where water is required for product washing, dust suppression, drilling, human consumption, and numerous other uses. Sound water management in mining is therefore required to provide a reliable water supply, especially when mine production is rapidly growing and competition of water use with other sectors is increasingly intensifying. However, highly variable climate conditions, leading to excess or deficient water on mining sites, create difficulties in providing reliable water supply to mining operations. It is crucial to include climatic variability in scientific decision-making tools to identify effective mine water management strategies.
\end{abstract}

Given the high uncertainties in long-term climate predictions, a strategy evaluation approach that can select reliable or robust strategy options under a set of possible future climates is promising. Growing evidences have identified the existence of low-frequency climate signals. However, the statistics of the climate signals are usually poor represented in commonly used climate-foresting models, such as general circulation models. This paper first presents a long-term daily rainfall and evaporation prediction model based on the observation of low-frequency climate signals, such as El Nino Southern Oscillation and Interdecadal Pacific Oscillation. Here, a Markov switching model is built to mimic the regime-like behavior of long-term annual climatic time series. Daily climatic patterns are selected from a historical year that is the most similar to the prediction year. Then a Monte Carlo method is used to create a large number of possible future climate sequences, each of which is a discrete Markov chain and represents a type of possible future climate condition. A future climate sequence can drive a process-based model of mine water use and simulate the impacts of an alternative management strategy. Thus, a set of alternative strategies can be evaluated under the different climate patterns. Finally, under each climate pattern, the performances of alternative strategies are produced. The information allows mine water managers to select reliable management options with desirable performance outcomes under particular climate patterns. The results highlight that the proposed method can identify effective management strategies. The strategies that are evaluated from a large number of climate patterns can lead to greater stakeholder acceptance.

Keywords: risk assessment, reliable water supply, water resources management, mining, Markov switching model 


\section{INTRODUCTION}

Water is essential in mine production and required for product washing, dust suppression, drilling, human consumption, and numerous other uses. In Australia, sound water management in mining is therefore required to provide a reliable water supply, especially when mine production is rapidly growing, competition of water use with other sectors is increasingly intensifying, and surface water and groundwater are already highly allocated (Gao et al., 2013b). However, managing mine water resources is a major challenge and closely associated with mine production and profitability. It could be more challenging if resource managers do not have scientific tools to assess the impacts of management strategies (Gao and Hailu, 2013). Water resource managers may need to deal with scarcity of water at one time and excesses in flooding at the other. Scarcity and excess of water are often related to climatic extremes. It is crucial to include climatic variability in scientific decision-making tools to identify effective mine water management strategies. The impacts of possible management actions in highly dynamical future climates need to be assessed.

Growing evidences have identified the existence of low-frequency climate signals (Akintug and Rasmussen, 2005). In Queensland, Australia, El Nino Southern Oscillation (ENSO) might be the single most important factor that affects rainfall variability. ENSO links ocean circulation and climate variation, and occurs with irregular intervals of 4-7 years. During the ENSO period, other phenomena, such as La Nina and Interdecadal Pacific Oscillation (IPO), may reinforce ENSO and lead to climate extremes. For example, in the 10 months since mid-2010, the developing ENSO La Nina was reinforced by the IPO leading to the highest sea surface temperatures off Australia's northeast coast and the highest rainfall totals in southeast Queensland on record.

Unfortunately, the statistics of the climate signals are usually poor represented in commonly used climateforesting models, such as general circulation models. This paper presents a Markov switching model-based prediction approach of future climate variability. Markov switching models or hidden Markov models have been applied to model climatic time series that are affected by quasi-cyclic climatic processes such as ENSO and IPO. However, these applications usually limit on annual long-term or daily short-term climatic process modeling. Here, a Markov switching model is built to mimic the regime-like behavior of long-term annual climatic time series. Daily climatic patterns are selected from a historical year that is the most similar to the predicted year. Then the daily climatic data drive a process-based simulation model of mine water use and produce the impacts of an alternative management strategy. Finally, the reliability of the alternative strategy is evaluated under a certain number of Monte Carlo runs, each of which is a discrete Markov chain and represents a kind of possible future climate sequence.

The rest of paper is organized as follows. Section 2 depicts the climate-driven approach for evaluating mine water management strategies under highly dynamical future climates. A coal mine is used as a case study in Section 3. Results from the case study are presented in Section 4. The final section concludes the paper.

\section{METHODS}

Precise long-term climate predictions seem impossible. A strategy evaluation model that can select reliable or robust strategy options under a set of possible future climates is promising. The proposed approach aims at modeling possible future climates and evaluating strategy alternatives under them. The approach includes a daily climate prediction model, a process-based simulation model of mine water use, and a Monte Carlo analysis.

\subsection{Daily rainfall and evaporation prediction model}

In meaningful planning to adopt a new management strategy, it is important to understand its performance, for instance reliability, in the condition of future possible climate variability. Given that low-frequency climate signals has been identified in Australian climatic time series data at annual and decadal timescales, we firstly represent future long-term persistence in climatic data (time series rainfall data in this paper) by assuming that the annual time series can switch between a few climate regimes or states. Then we select daily climatic patterns from a historical year that is the most similar to the predicted year. Finally, we map the annual data of the predicted year into daily time series data based on the selected daily climatic patterns. 
The model we used to represent state sequence of annual climatic data as a Markov chain is called Markov switching model. We assume climate regime of each year is switching between $N$ state variables and annual rainfall is associated with the climate regime. The evolution of these state variables is determined by a matrix of transition probabilities to each state variable switched from the current one. The transition probability matri

$\mathrm{x} P$ is

descr

$$
P=\left[p_{a b}\right], \quad p_{a b}=\operatorname{pr}\left(c_{t}=b \mid c_{t-1}=a\right)
$$

ibed in eq. (1).

where $p_{a b}$ is a transition probability that current state variable is $b$, given that the previous state variable is $a$ $(a, b \in(1,2 \ldots, N))$, and $c_{t}$ is the state variable in year $t$.

Following Thyer and Kuczera (2000), we assume the observed variable $x_{t}$ follows a normal distribution with mean $\mu_{c_{t}}$ and variance $\sigma_{c_{t}}$ that are specific to state $c_{t}$. For a given sequence of observations, the appropriate number of states is usually unknown and needs to be selected based on objective criteria. The most common criteria include Akaike information criterion (AIC) and Bayesian information criterion (BIC) (Zucchini and Mac

Dona

ld,

$$
A I C=-2 \log L+2 p
$$

2009). AIC and BIC are defined in eqs. (2) and (3).

where $\log L$ is the $\log$-likelihood of the fitted model and p denotes the number of parameters of the model.

wher

$$
B I C=-2 \log L+p \log T
$$

e $\log L$ and $p$ are as for AIC, and $T$ represents the number of observations.

Because there are a relatively high number of parameters in Markov switching model, the method of maximum likelihood is more likely to produce good estimates than the method of moments (Akintug and Rasmussen, 2005). The EM (Expectatioin-Maximalization) algorithm (Zucchini and MacDonald, 2009) is employed find maximum likelihood estimates of model parameters. Given estimated state $c_{t}$, associated mean $\mu_{c_{t}}$ and variance $\sigma_{c_{t}}, x_{t}$ can be generated as eq. (4)

$$
x_{t}=\mu_{c_{t}}+\sigma_{c_{t}} \varepsilon_{t}
$$

where $\varepsilon_{t} \sim N(0,1)$ is a standard normal variable.

The next step is to convert annual observation $x_{t}$ into daily observations in year $t$. Year $\tilde{t}$ is selected from historical time series records in terms of the most similarity indicator, for example the nearest annual rainfall volume. Let $X_{D}=\left(x_{t}^{1}, \ldots, x_{t}^{d}, \ldots, x_{t}^{D}\right)$ be the vector of daily time series observations in year $t$. Daily climatic data

$x_{t}^{d}$ can

be

$$
x_{t}^{d}=x_{\tilde{t}}^{d} \cdot \frac{x_{t}}{x_{\tilde{t}}}
$$

ted as eq. (5)

where $x_{\tilde{t}}$ is annual observation and $x_{\tilde{t}}^{d}$ is daily observation in day d both in year $\tilde{t}$.

\subsection{A process-based mine water simulation model}

We also developed a climate-driven model for simulating mine water uses in different strategies. The model abstracts the water system in a mine site as a number of objects, such as water stores, water tasks, a treatment plant, water inputs, and water outputs. A water store is a water storage facility on site. A water task represents a type of activities that use water for a particular purpose. Water input and output objects stand for water inputs to the mine water system from exteriors and water outputs from the site to external destinations, 
respectively. A treatment plant is a facility object that is responsible for improving water quality. A water use process was further developed to simulate dynamics of water quantity and quality in the objects. The impacts of a water management strategy can be simulated by the model, such as failure rate of supplying water tasks, raw water use per mine production, water use efficiency, water use cost, unregulated discharge, and risks associated with water quantity and quality. The model provides flexibility in exploring complicated water use strategies between raw and worked water stores. We refer readers to Gao et al. (2013a) for more details of the process-based simulation model.

\subsection{Monte Carlo analysis}

A Monte Carlo analysis was used to account for variations in the amount of rainfall and evaporation. The daily rainfall and evaporation prediction model is used in a Monte Carlo simulation as follows: for each of $M$ Monte Carlo draws ( $M$ is a large number, for example 10,000), annual rainfall state $c_{t}$ is assigned by random draw with transition probabilities given previous year state $c_{t-1}$, as shown in eq. (1). The annual volume of rainfall is generated by a random normal draw from a normal distribution with mean $\mu_{c_{t}}$ and variance $\sigma_{c_{t}}$. Finally the target performance indicators are accumulated over $K$ simulation years and $M$ Monte Carlo draws. Additionally, a small set of "extreme" climates can be selected from the $M$ Monte Carlo draws to assess the performance of alternative strategies under extreme climates.

\section{CASE STUDY}

We applied the proposed approach to the assessment of strategy reliability in an open-cut coal mine in Bowen Basin, Queensland, Australia. Three types of water tasks are identified in the case study: coal handling and preparation plant (CHPP), dust suppression, and underground operations. The data for parameterizing the process-based simulation model is reported by Cote et al. (2008) as mine 3. A climate database was established by integrating rainfall and evaporation (ET) data for the study area downloaded from a selected weather station from SILO (http://www.longpaddock.qld.gov.au/silo/datadrill/index.php) website which provides an enhanced climate data bank hosted by The Science Delivery Division of the Department of Science, Information Technology, Innovation and the Arts (DSITIA), Queensland Government.

Then a set of management strategies are designed and assessed under possible future climates. First, we have a baseline strategy that represents business-as-usual situation. In the baseline strategy, the model is calibrated in terms of reported indicators during the period from $1^{\text {st }}$ July 1977 to $30^{\text {th }}$ June 2007 . The calibration performance is reported in (Gao et al., 2013a). Based on the calibrated model, we develop and compare different mine water use strategies, including different combinations of store use sequences and whether a treatment plant is employed. The strategies related sequences of store use considered in this paper include: worked-first, one-store, and optimal-mixture. The worked-first strategy first use worked water store to supply water tasks. Only after using up water in the worked water store, one can use the raw water store. In the onestore strategy, one needs to use water from the worked store; however, if the water is not acceptable, one can use the raw water store. The strategy cannot use water from the two stores simultaneously. The optimalmixture strategy optimises water mixture from two stores for minimal water use cost. The above three strategies are undertaken for two treatment levels (employing and not employing a treatment plant).

\section{RESULTS AND DISCUSSION}

We first model the stochastic nature of annual rainfall using historical data from water year 1992-2012. To determine the optimal number of states of the Markov switching model, we considered models of 2, 3, 4, and 5 states. The log-likelihood, AIC, and BIC values of the five models are given in Table 1. In terms of AIC and BIC, a 2-state Markov switching model is the best choice.

The EM algorithm is used to produce the estimates of the 2-state model parameters. The initial state and state transition are assumed to follow multinomial distributions. The estimated transition probability matrix, as well as means and variances associated with each state are shown in Table 1. The resulting model illustrates that two well-separated states, one with low (L) and one with high (H)
Table 1. Comparison of (stationary) Markov switching models (MSM) by log-likelihood, AIC and BIC.

\begin{tabular}{cccc}
\hline Model & $-\boldsymbol{\operatorname { l o g }} \boldsymbol{L}$ & $\mathbf{A I C}$ & $\mathbf{B I C}$ \\
\hline 2-state MSM & 132 & 279 & 286 \\
3-state MSM & 126 & 281 & 295 \\
4-state MSM & 123 & 291 & 314 \\
5-state MSM & 112 & 292 & 326 \\
\hline
\end{tabular}


annual rainfall responses. The transition probabilities indicate relatively stable states: the probabilities of remaining in one state are 0.63 and 0.9 respectively.

20-year climatic time series from 2013 to 2032 are predicted using the proposed approach. Using eq. (1) and transition probability matrix in Table 2 , one can work out a chain of climatic states for the 20 years. Then, in terms of eq. (4) and Table 2, a chain of annual climatic time series are generated. The historical rainfall data from 1889 to 2012 water year is used to build a set of daily climatic time series patterns. Further, a chain of daily climatic time series are produced according to eq. (5). By repeating the above steps for 10,000 Monte Carlo draws, we obtained 10,000 possible daily climatic time series for the 20 years.

Each obtained daily climatic time series can drive a single run of mine water use simulation for 20 years. Next, we present the simulation results under baseline and the other six strategies (worked-first, one-store, and optimal-mixture with and without employing a desalination treatment). Mine water use in each strategy was simulated under 10,000 possible climatic times series. Four indicators were selected to evaluate the seven mine water strategies: water supply reliability, water use cost, raw water use, and unregulated discharge. The water supply reliability represents a success rate of water supply under 10,000 climatic conditions, and is calculated as a ratio of the number of times to offer acceptable water to total demand times. The water use cost, the raw water use, and the unregulated discharge are measured in the condition of per Megaton coal production. The unregulated discharge refers to overflow from the worked water store to the raw water store. The four performance indicators of the seven strategies are shown in Table 3.

Table 3. Performance indicators of mine water management strategies.

\begin{tabular}{ccccc}
\hline Strategy & Reliability & $\begin{array}{c}\text { Cost } \\
(\mathbf{\$ M / M t})\end{array}$ & $\begin{array}{c}\text { Raw water } \\
\text { use (ML/Mt) }\end{array}$ & $\begin{array}{c}\text { Unregulated } \\
\text { discharge (ML/Mt) }\end{array}$ \\
\hline baseline & $94.95 \%$ & 0.83 & 487 & 1.17 \\
worked-first & $1.35 \%$ & 0.54 & 313 & 0.14 \\
worked-first plus treatment & $1.71 \%$ & 0.55 & 313 & 0.53 \\
one-store & $98.34 \%$ & 0.91 & 546 & 15.33 \\
one-store plus treatment & $98.44 \%$ & 0.87 & 511 & 6.47 \\
optimal-mixture & $98.63 \%$ & 0.56 & 304 & 0 \\
optimal-mixture plus treatment & $98.76 \%$ & 0.51 & 282 & 0 \\
\hline
\end{tabular}

Table 3 shows that the two worked-first strategies cannot ensure a secure water supply, as a result of that water quality in the worked water store is not acceptable during the most of simulation time. Therefore, they are removed from strategy candidates. The two one-store strategies and two optimal-mixture strategies lead to high reliability in mine water supply under 10,000 possible future climate chains. Compared with the baseline strategy, the one-store strategies increase supply reliability by more than $3 \%$. However, the increase in supply reliability is counteracted by rises in supply cost, raw water use, and unregulated discharge. In terms of the four performance indicators, the two optimal-mixture strategies perform the best among all strategies. The two strategies gain additional 3.5\% reliability level, reduce more than $32 \%$ of the water cost, save over $37 \%$ of the raw water use, and eliminate unregulated discharge, compared to the baseline strategy. Employing a desalination plant is found helpful to raise supply reliability, reduce raw water use, and decrease supply cost.

With the simulation outcomes under 10,000 possible future climate conditions, we can further evaluate mine water management strategies in a particular climate pattern. Table 4 demonstrates supply reliabilities of five water use strategies in high rainfall years, low rainfall years, $50^{\text {th }}$ percentile in continuous low rainfall years, and $99^{\text {th }}$ percentile in continuous low rainfall years. 
Gao et al., Strategic water management for reliable mine water supply under dynamical climates

Table 4. Supply reliabilities of mine water management strategies in different climate patterns.

\begin{tabular}{ccccc}
\hline Strategy & $\begin{array}{c}\text { High } \\
\text { rainfall } \\
\text { years }\end{array}$ & $\begin{array}{c}\text { Low } \\
\text { rainfall } \\
\text { years }\end{array}$ & $\begin{array}{c}\mathbf{5 0}^{\text {th }} \text { percentile in } \\
\text { continuous low } \\
\text { rainfall years }\end{array}$ & $\begin{array}{c}\mathbf{9 9}^{\text {th }} \text { percentile in } \\
\text { continuous low } \\
\text { rainfall years }\end{array}$ \\
\hline baseline & $96.36 \%$ & $89.38 \%$ & $97.78 \%$ & $71.77 \%$ \\
one-store & $98.80 \%$ & $96.53 \%$ & $98.56 \%$ & $97.22 \%$ \\
one-store plus treatment & $98.89 \%$ & $96.64 \%$ & $98.67 \%$ & $97.27 \%$ \\
optimal-mixture & $99.06 \%$ & $96.93 \%$ & $98.81 \%$ & $97.71 \%$ \\
optimal-mixture plus treatment & $99.19 \%$ & $97.05 \%$ & $98.94 \%$ & $97.79 \%$ \\
\hline
\end{tabular}

In terms of supply reliability, the risk is larger in low rainfall years than that in high rainfall years. Poor water management in low rainfall years can result in running out of water, which will cost money in lost production. As shown in Table 4, each management strategy performs at higher supply reliability level in high rainfall years than low rainfall years. We further assess the five management strategies in a relatively extreme climate pattern: $99^{\text {th }}$ percentile in continuous low rainfall years (in the climate chains that have continuous six low rainfall years). The two one-store strategies and the two optimal-mixture strategies demonstrate a strong robustness towards the extreme climate pattern and their reliabilities decrease only about $1 \%$, compared with those in the $50^{\text {th }}$ percentile in continuous low rainfall years (in the climate chains that have continuous two low rainfall years). In contrast, the supply reliability of the baseline strategy plummets by $26 \%$ to $71.77 \%$ in the extreme climate pattern from $97.78 \%$ in the $50^{\text {th }}$ percentile in continuous low rainfall years.

\section{CONCLUSIONS}

Strategy evaluation plays a prominent role in managing mine water resources under highly variable climates. However, key methodological challenges remain due to the fact that precise long-term climate predictions are extremely difficult. This study proposes a new strategy evaluation method for identifying sound management strategies. We first build a daily rainfall and evaporation prediction model based on the observation of lowfrequency climate signals. Then a Monte Carlo method is used to create a large number of possible future climate sequences, under which a set of alternative strategies are evaluated. Finally, under each climate pattern, the performances of alternative strategies are produced, allowing mine water managers to select reliable management options with desirable performance outcomes under particular climate patterns. The results highlight that the proposed method can identify effective management strategies from those are not. The strategies that are evaluated from a large number of climate patterns can lead to greater stakeholder acceptance. Future work includes improving daily rainfall estimation by adding climate change to the climate variability, for example a shift in the daily rainfall distribution.

\section{ACKNOWLEDGMENT}

This work is supported by the Australian Coal Association Research Program as project 51088 and CSIRO Water for a Healthy Country Flagship as appropriation research project "Strategic Water Management in Mining”. A coauthor (Dr. Xiangfeng Zhang) would like to express her gratitude to the support of the Innovation Program of Shanghai Municipal Education Commission (NO.13YZ139).

\section{REFERENCES}

Akintug, B., Rasmussen, P.F., (2005). A Markov switching model for annual hydrologic time series. Water Resources Research, 41(9), W09424.

Côte, C.M., Moran, C.J., Gozzard, E., Craven, A., Shih, J., (2008). Understanding Leading Practice in Water Management. ACARP report C16035, http://www.acarp.com.au/search.aspx?\&qS=C16035.

Gao, L., Barrett, D., Chen, Y., Zhou, M., Cuddy, S., Paydar, Z., Renzullo, L., (2013a). A process-based simulation model for strategic mine water management, 20th International Congress on Modelling and Simulation: Adelaide, South Australia.

Gao, L., Connor, J., Doble, R., Ali, R., McFarlane, D., (2013b). Opportunity for peri-urban Perth groundwater trade. Journal of Hydrology, 496, 89-99.

Gao, L., Hailu, A., (2013). Identifying preferred management options: An integrated agent-based recreational fishing simulation model with an AHP-TOPSIS evaluation method. Ecological Modelling, 249, 75-83. 
Gao et al., Strategic water management for reliable mine water supply under dynamical climates

Thyer, M., Kuczera, G., (2000). Modeling long-term persistence in hydroclimatic time series using a hidden state Markov Model. Water Resources Research, 36(11), 3301-3310.

Zucchini, W., MacDonald, I.L., (2009). Hidden Markov Models for Time Series: An Introduction Using R. CRC Press, Taylor \& Francis Group, Florida, USA. 